\title{
Aplysia Neurons as a Model of Alzheimer's Disease: Shared Genes and Differential Expression
}

\author{
Nicholas S. Kron ${ }^{1}$ [D Lynne A. Fieber ${ }^{1}$
}

Received: 5 August 2021 / Accepted: 18 September 2021 / Published online: 18 October 2021

(c) The Author(s) 2021

\begin{abstract}
Although Alzheimer's disease (AD) is the most common form of dementia in the United States, development of therapeutics has proven difficult. Invertebrate alternatives to current mammalian AD models have been successfully employed to study the etiology of the molecular hallmarks of AD. The marine snail Aplysia californica offers a unique and underutilized system in which to study the physiological, behavioral, and molecular impacts of AD. Mapping of the Aplysia proteome to humans and cross-referencing with two databases of genes of interest in AD research identified 898 potential orthologs of interest in Aplysia. Included among these orthologs were alpha, beta and gamma secretases, amyloid-beta, and tau. Comparison of age-associated differential expression in Aplysia sensory neurons with that of late-onset AD in the frontal lobe identified 59 ortholog with concordant differential expression across data sets. The 21 concordantly upregulated genes suggested increased cellular stress and protein dyshomeostasis. The 47 concordantly downregulated genes included important components of diverse neuronal processes, including energy metabolism, mitochondrial homeostasis, synaptic signaling, $\mathrm{Ca}^{++}$regulation, and cellular cargo transport. Compromised functions in these processes are known hallmarks of both human aging and AD, the ramifications of which are suggested to underpin cognitive declines in aging and neurodegenerative disease.
\end{abstract}

Keywords Beta-amyloid $\cdot$ Tau $\cdot$ Neuroinflammation $\cdot$ Invertebrate model

\section{Introduction}

Aging in humans is often accompanied by progressive declines in cognitive capabilities that can result in the inability to perform basic tasks, known clinically as dementia (Weller and Budson 2018). By far the most common of these dementias is Alzheimer's disease (AD), accounting for up to $80 \%$ of dementia cases (Crous-Bou et al. 2017). In addition to neurodegeneration, $\mathrm{AD}$ is distinguished from other dementias by the presence of two types of protein aggregates, amyloid-beta $(A \beta)$ plaques and hyperphosphorylated tau protein neurofibrillary tangles, in addition to neurodegeneration (Jack et al. 2018). As of 2014, despite more than 30 years of clinical research, only five drugs had been identified as sufficiently safe and effective for international marketing approval, and these provide mostly modest clinical

Nicholas S. Kron

n.kron@umiami.edu

1 Department of Marine Biology and Ecology, Rosenstiel School of Marine and Atmospheric Science, University of Miami, 4600 Rickenbacker Cswy, Miami, FL 33149, USA effects (Schneider et al. 2014). The difficulty in studying this illness in living patients coupled with a complex etiology are major hurdles to the study of $\mathrm{AD}$ and development of effective drugs to treat it.

One factor that may contribute to the difficulty in $\mathrm{AD}$ research thus far is the inability of many model systems to recapitulate the complex nature of the disease. Medina and Avila (2014) assert that an ideal AD model should be able to integrate the genetic, environmental, and aging factors that contribute to $\mathrm{AD}$ disease progression. Unfortunately, many current models often address only one factor in isolation (Medina and Avila 2014). However, invertebrate models offer possible alternatives in modeling the complex states which give rise to AD (Calahorro and Ruiz-Rubio 2011; Fernandez-Funez et al. 2015; Sharma et al. 2017). Not only are these models often faster, cheaper, and in line with ethical efforts to reduce the use of vertebrates in research, but they also offer unique investigative techniques or more amenable environments for study when compared to vertebrate models (Alexander et al. 2014; Gotz and Ittner 2008; Link 2005; Moloney et al. 2010; Prussing et al. 2013; Sharma et al. 2017; Surguchov 2021). 
Invertebrate models have provided an alternative approach to traditional mammalian models and have been instrumental in elucidating key components of disease progression in $\mathrm{AD}$ and $\mathrm{AD}$-related dementias (ADRD). The tractability of behavioral phenotypes and molecular techniques in Drosophila melanogaster and Caenorhabditis elegans have made these two popular invertebrate models effective tools in investigating disease mechanisms of AD and ADRD and for drug target discovery in AD and ADRD. For example, the molecular basis for $A \beta$ and tau aggregation and toxicity were elucidated via these model systems (Fernandez-Funez et al. 2015; Hannan et al. 2016).

An underutilized model system in which to study AD and ADRD is the marine gastropod Aplysia californica (Aplysia). Among the preeminent models for learning, Aplysia is a well-described neural model ideal for the integrated study of learning and behavior at the molecular, cellular, neural-circuit, and whole organism levels (Baxter and Byrne 2006; Carew et al. 1983; Castellucci et al. 1970; Cleary et al. 1998; Kindy et al. 1991; Klein et al. 1982; Kupfermann 1974; Moroz 2011; Moroz et al. 2006). Due to an annual life span and a well-mapped nervous system, Aplysia has also proven to be an excellent model for investigating the effects of aging on learning, cognitive function, and neuronal physiology (Bailey et al. 1983; Hallahan et al. 1992; Kempsell and Fieber 2014, 2015a, b, 2016; Papka et al. 1981; Peretz et al. 1984; Rattan and Peretz 1981; Srivatsan and Peretz 1996). Molecular studies of the effects of aging on the transcriptomes of sensory neurons ( $\mathrm{SN}$ ) revealed similar aging signatures as those of other animals, including metabolic, proteostatic, and neuro-synaptic impairments similar to those that also occur in AD and ADRD (Greer et al. 2019; Greer et al. 2018; Kron et al. 2020). Furthermore, transcriptomic profiling of individually identified giant neurons in Aplysia have allowed for the investigation of the effects of aging on specific neurons (Kadakkuzha et al. 2013; Moroz and Kohn 2010, 2013). As a powerful neural aging model, Aplysia offers a unique system in which to study AD and ADRD in the context of the greatest risk factor for AD development.

Previously, cultured Aplysia neurons have been demonstrated to recapitulate $\mathrm{AD}$-like taupathies when transfected with mutant human tau (Shemesh and Spira 2010). These neurons were subsequently used to investigate the efficacy of a potential AD therapeutic (Shemesh and Spira 2011). Similarly, exposure of cultured neurons from closely related $A$. kurodai to mutant human $\mathrm{A} \beta$ elucidated the inhibitory effects of $A \beta$ on GABA-induced chloride currents (Sawada and Ichinose 1996). Furthermore, cultured A. kurodai sensory-motor neuron co-cultures were used to investigate the formation and deleterious effects of cofilin-actin rods, hypothesized to be the precursors to the protein aggregates that typify AD and ADRDs like Parkinson's disease and amyotrophic lateral sclerosis, via overexpression of the native cofilin gene (Jang et al. 2005). Together these studies highlight the applicability of the Aplysia model system to allow for the study of AD in the context of behavior, genetics, and aging.

In this study, we further demonstrate that Aplysia offers a suitable model for the study of AD and ADRD by combing the Aplysia genome for potential orthologs of genes of interest in AD and ADRD. We also compare available molecular aging data of Aplysia sensory neurons (SN) to those of lateonset AD (LOAD) to demonstrate the capacity of Aplysia neurons to naturally recapitulate the preconditions and risk factors that are believed to contribute to $\mathrm{AD}$ development in human aging.

\section{Methods}

\section{Aplysia Genome Annotation}

The RefSeq proteome for the latest Aplysia genome build (AplCal3.0) was downloaded from the NCBI FTP site (https://ftp. ncbi.nlm.nih.gov/genomes/all/annotation_releases/6500/101/ GCF_000002075.1_AplCal3.0/). The human UniProt proteome (UP000005640) was downloaded from the UniProt website (https://www.uniprot.org/proteomes/UP000005640) and used to construct a local blast database using the BLAST + command line tool (version 2.6.0; Camacho et al. 2009). The Aplysia proteome was then blasted against the human proteome, selecting only the top hit with an e value of $\leq 0.001$. These Aplysia-tohuman protein annotations were then imported into the $\mathrm{R}$ statistical environment and further annotated to the transcript and gene level for Aplysia using the latest gene feature format (gff, version 1.21) file available for AplCal3.0 at the NCBI FTP site. Human proteins were annotated to the gene level by mapping UniProt protein identifiers to human gene symbols using the org.Hs.eg.db R package (Carlson 2019; R Core Team 2013; Wickham et al. 2019).

\section{Overlap with Alzheimer's Genes of Interest}

The putative Aplysia-human orthologs generated in the previous section were then intersected with two genome-wide association meta-analysis-derived gene sets of Alzheimer'sassociated genes: Alzgset (Hu et al. 2017) and AlzGene (Bertram et al. 2007).

\section{Comparison of Aplysia Sensory Neuron Aging and LOAD in the Frontal Lobe}

Gene sets previously identified as differentially expressed in aging in Aplysia SN (Greer et al. 2018; Kron et al. 2020) were collected and compared with genes identified as 
differentially expressed in LOAD via meta-analysis of six different frontal lobe data sets ( $\mathrm{Li}$ et al. 2015). In their meta-analysis, Li et al. (2015) considered genes that were identified as significant and had concordant direction of expression change in at least five of the six data sets used. In our comparison with Li et al. (2015), we selected all genes marked as DE and exhibited concordant expression direction in at least two of the three Aplysia data sets (PVC from Greer et al. 2018, and PVC and BSC from Kron et al. 2020), and exhibited concordant expression direction in at least five human data sets from Li et al. (2015).

\section{Results}

\section{Aplysia Proteome Annotation}

Out of 26,658 unique proteins in the Aplysia RefSeq database, 20,495 proteins mapped to 9116 unique UniProt identifiers, equaling on average 2.3 Aplysia proteins per human protein. Each UniProt protein is mapped to one gene in the UP000005640 reference proteome; thus the 20,500 Aplysia proteins were mapped to $\sim 9000$ human genes.

Among these putative orthologs were several human genes involved in AD and ADRD. An ortholog of amyloid precursor protein (APP) was identified in Aplysia previously, and here we identified two potential APP orthologs (Moroz and Kohn 2010). Similar to Drosophila, but unlike C. elegans, we identified putative Aplysia orthologs of both beta-secretase 1 (BACE1) and all components of the gamma-secretase complex: presenilin (PSEN), nicastrin (NCSTN), presenilin enhancer 2 (PSENEN), and two putative orthologs of anterior pharynx-defective 1 (APH1A). We also identified several potential Aplysia orthologs to the primary alpha secretase A disintegrin and metalloproteinase (ADAM) family members including three orthologs of ADAM10, two orthologs of ADAM12, and seven orthologs of ADAM17. Two potential orthologs of the tau protein gene MAPT were also identified.

Of interest in Parkinson's disease, six potential orthologs of leucine-rich repeat kinase 2 (LRRK2/PARK8), along with putative orthologs of other Parkinson's disease-associated genes such as protein deglycase DJ-1 (PARK7/DJ-1), Parkin (PRKN), Parkin coregulated gene protein (PACRG), and synphilin (SNCAIP), were identified. However, a potential ortholog for alpha-synuclein (SNCA/PARK1) was not identified.

\section{Overlap with Alzgset and AlzGene}

Of the 9000 putative orthologs, 219 were present in Alzgset and 364 were present in AlzGene. Alzgset and AlzGene share 295 genes, of which 166 were among the $~ 9000$
Aplysia-human orthologs. Considering genes from either data set, a total of $418 \mathrm{AD}$ genes of interest with putative orthologs in the Aplysia genome were identified (Fig. 1). This corresponds to 1207 Aplysia transcripts from 898 Aplysia genes. As noted in the above section, orthologs of PSEN1, APP, and MAPT were present, along with several other $A \beta$ - and tau-associated proteins (Table 1). The full mapping is available in Supplemental Data 1.

\section{Comparison to LOAD Frontal Cortex Study}

Comparison of differential expression in three aging Aplysia $\mathrm{SN}$ data sets with a meta-analysis of six frontal cortex LOAD (FL LOAD) data sets identified 68 putative gene orthologs concordantly differentially expressed in at least five of the FL LOAD studies and two Aplysia data sets. Of these genes, 21 were concordantly upregulated and 47 concordantly downregulated. Commonly upregulated genes included cellular stress-induced genes such as ANKZF1, BTG1, DDIT4L, and SSR1, as well as elements of the proinflammatory toll/interleukin receptor signaling pathways such as MYD88, NFKBIA, MAP3K8, and BIRC3 (Fig. 2 and Table 2). Commonly downregulated genes were representative of diverse processes including synaptic vesicle dynamics (SYN2, EXOC8, NAPG, SVOP, ARF3), transport of cellular cargo (DCTN6, KIFAP3, RAB6A), energy metabolism

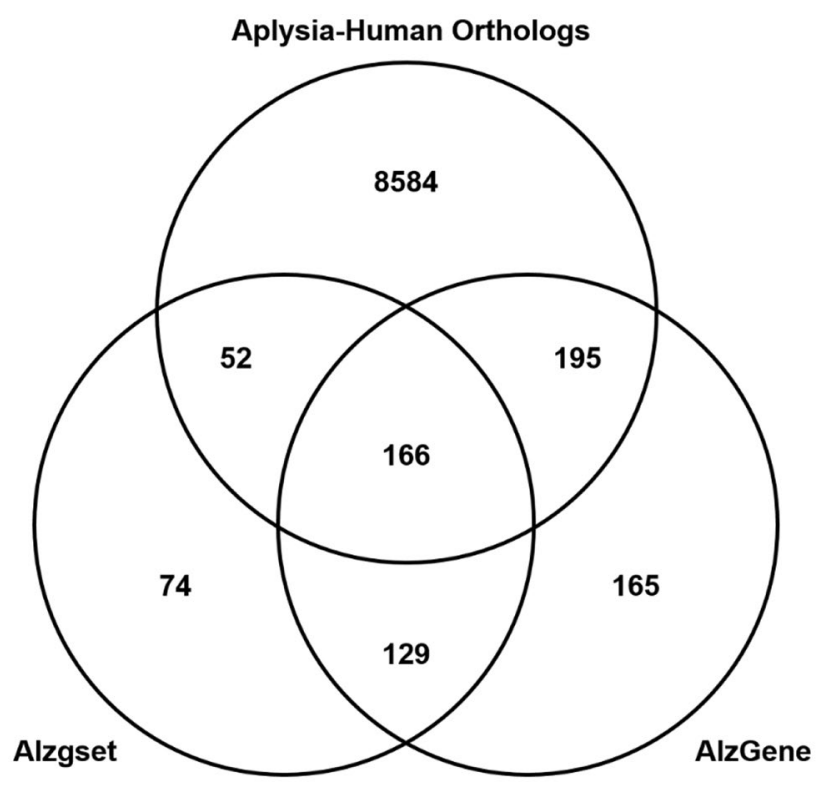

Fig. 1 Gene set overlap of putative human orthologs found in the Aplysia genome with Alzheimer's disease (AD)-associated gene databases Alzgset and AlzGene. Aplysia RefSeq proteins were mapped to the UniProt human protein database using the BLAST + command line tool. The two AD genes-of-interest data sets shared 295 genes. Of the more than 9000 Aplysia-human orthologs identified, 418 were present in either Alzgset, or AlzGene, or both. A smaller subset of 166 genes was identified as common to all three gene sets 


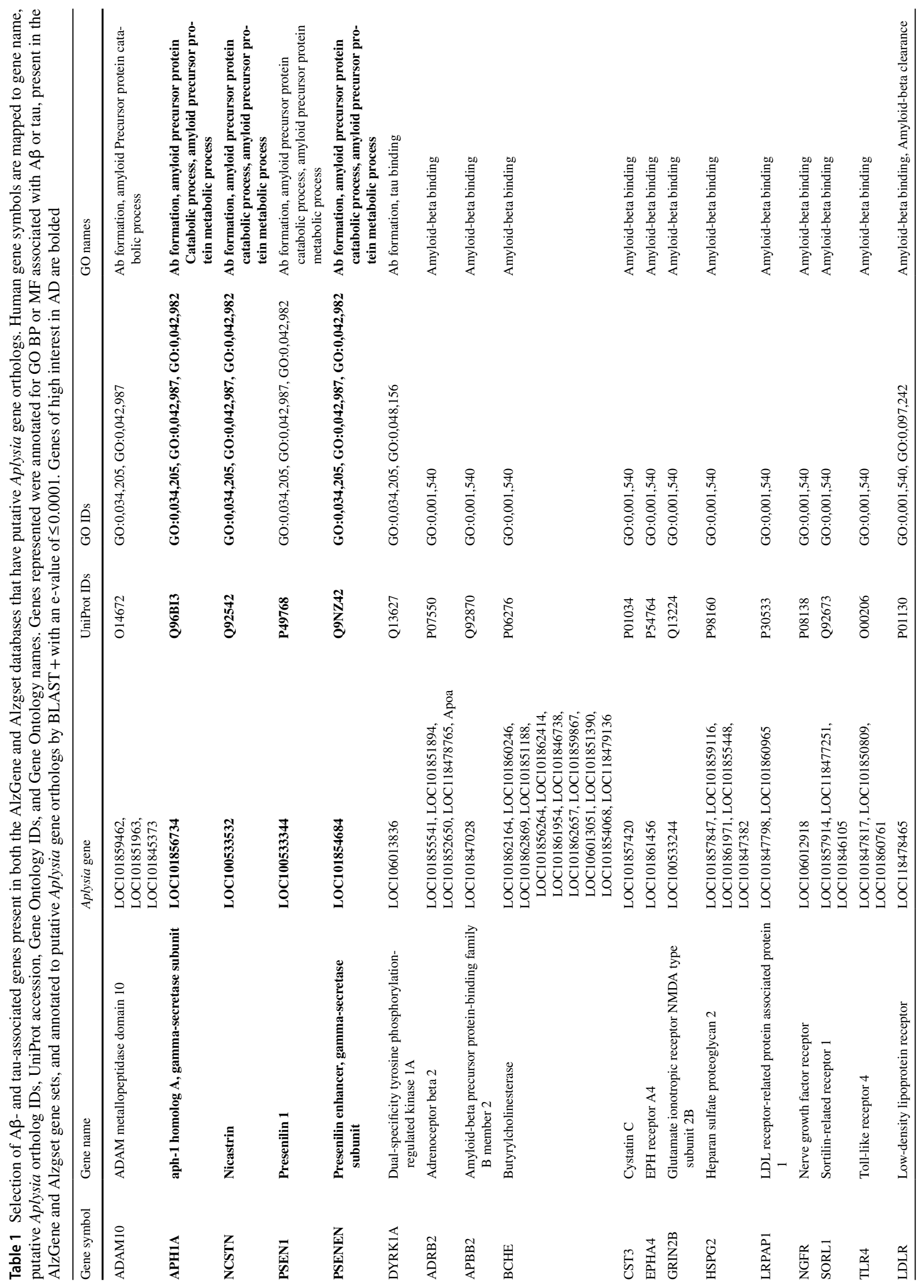




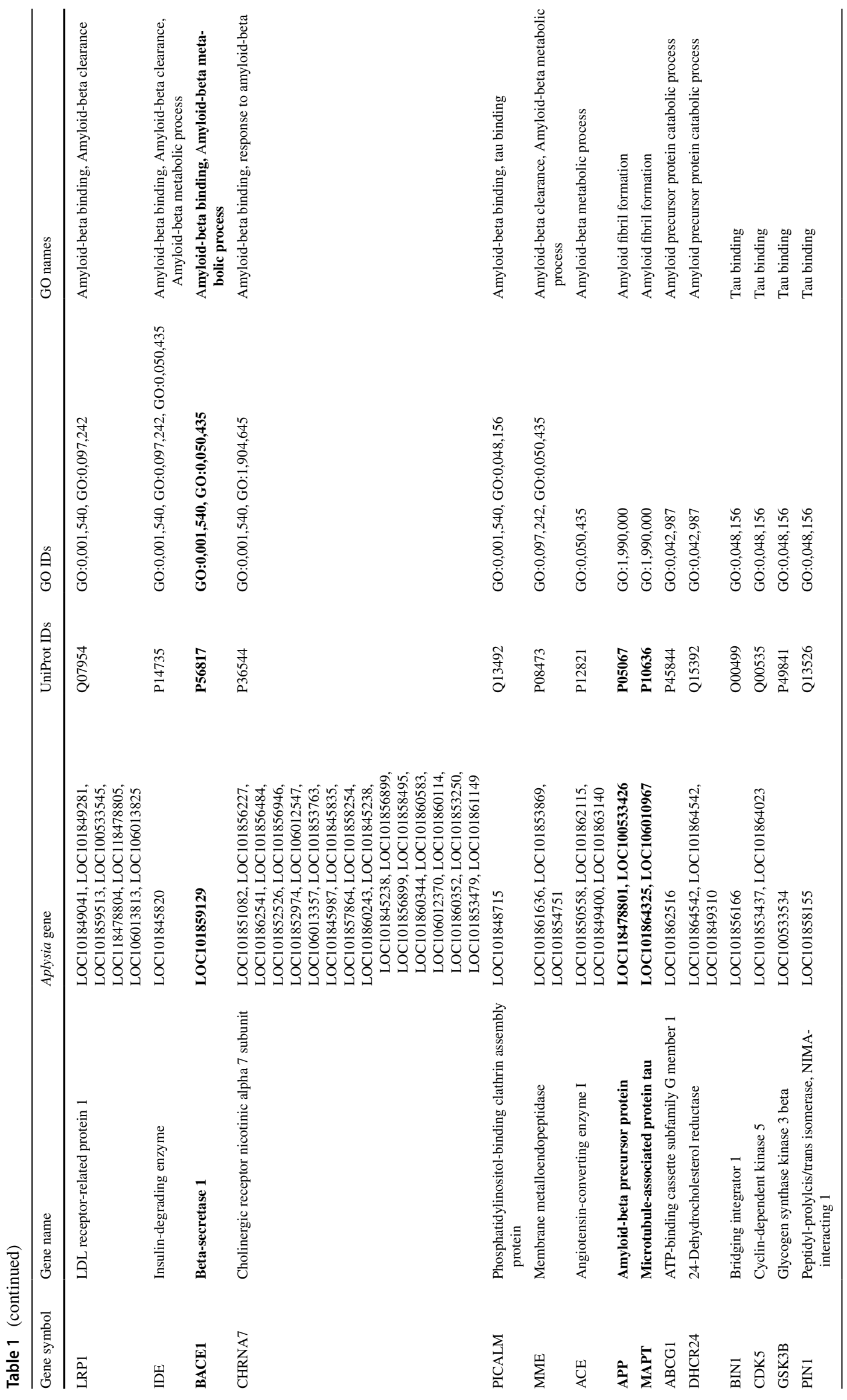




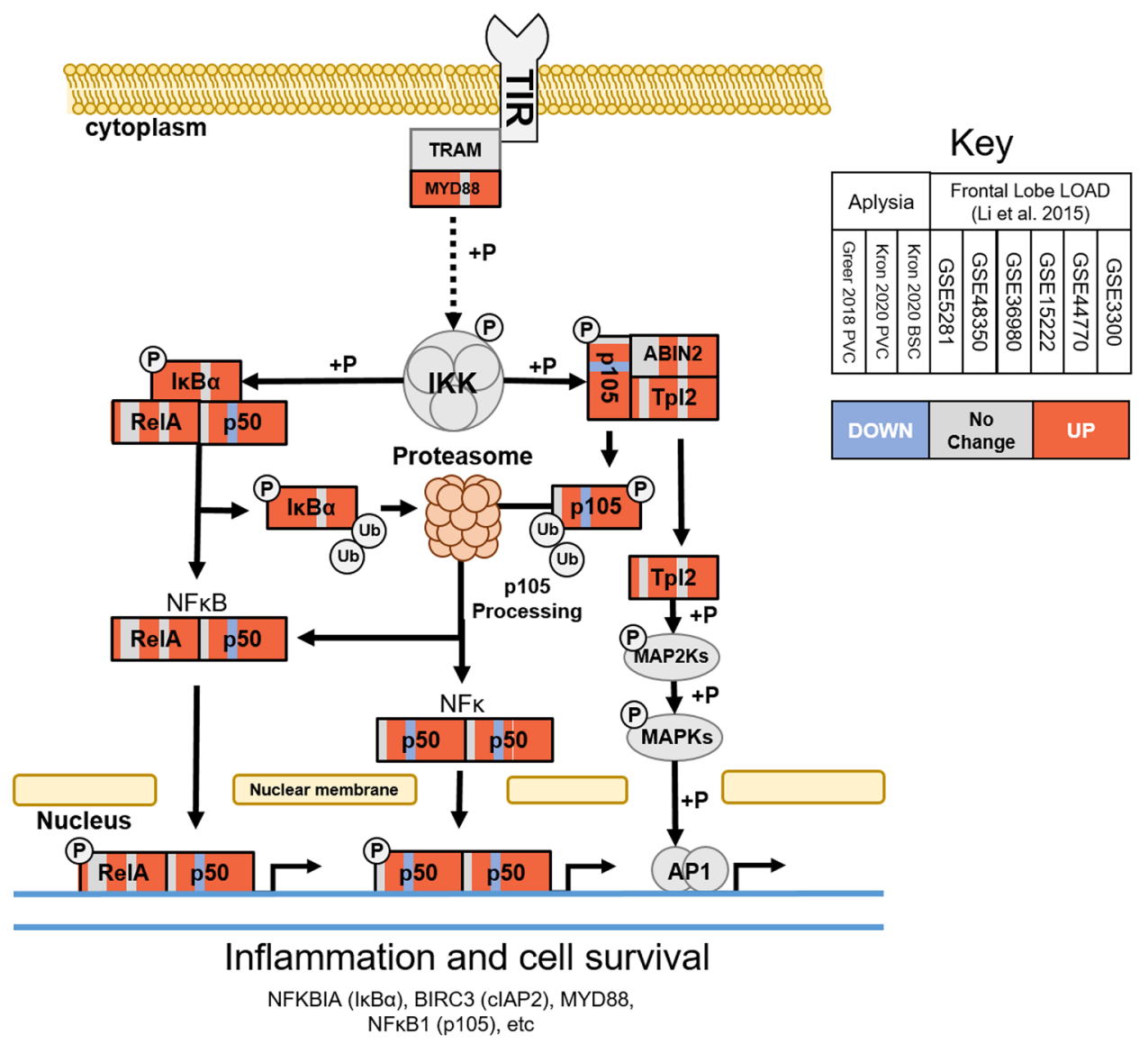

Fig. 2 Commonly upregulated orthologs in the toll/interleukin signaling pathway between Aplysia SN aging and FL LOAD. Each box represents a gene and is subdivided into nine sections corresponding to analyzed data sets; the first three are Aplysia SN data sets and the latter six are FL LOAD results from $\mathrm{Li}$ et al. (2015) as demarcated in the Key. Each section is colored to indicate whether the genes were upregulated (red), downregulated (blue), or exhibited no change (gray) in the corresponding data set. Solid line arrows represent a

(GOT1 and 2, MDH1, CYCS, NDUFV1, PCCB), cyclicAMP response element-binding protein (CREB)-mediated learning and memory (MAP2K1, PRKACA, CAMK4, ELAV4, Fig. 3) and mitochondrial homeostasis (GDAP1, TUSC2), among others (Table 3). The full gene list is available in Supplementary Data 2.

\section{Discussion}

In our screening of the Aplysia genome for orthologs to Alzheimer's-associated genes we identified 418 putative orthologs. Among these were orthologs of hallmark players in $\mathrm{AD}$ progression such as $\mathrm{A} \beta$ and tau.

The quintessential hallmark of $\mathrm{AD}$ is the formation of $A \beta$ plaques in the nervous system. $A \beta$ is a cleavage product of APP by the single protein beta secretase and direct interaction, while dotted lines signify indirect interaction via intermediaries. Example genes known to be upregulated by NF-kB that were common to both Aplysia SN aging and FL LOAD are listed at the bottom of the figure. Commonly upregulated genes include toll/ interleukin signaling adapter protein MYD88 and several components of the NF-kB signaling cascade (I $\kappa \mathrm{Ba}, \mathrm{NF}-\kappa \mathrm{B} 1$, TPL2), suggesting that increased proinflammatory signaling is a common feature of Aplysia SN aging and FL LOAD

the multi-protein gamma secretase enzymes. In contrast to beta and gamma secretases, alpha secretases process APP in a manner that does not produce $A \beta$. The alpha secretase ADAM10 has been demonstrated to compete with beta and gamma secretases for APP and confers protection from $A \beta$ accumulation and tau hyperphosphorylation (Peron et al. 2018; Yuan et al. 2017). While A $\beta$ plaques associated with AD in humans are not known to occur in invertebrates, endogenous orthologs of APP and associated secretases in Drosophila and C. elegans have been used to investigate the mechanisms by which these enzymes and cleavage byproducts function in normal and pathological conditions. This approach has shed light on the mechanisms of A $\beta$-related AD pathology, suggesting that Aplysia can be used similarly (Alexander et al. 2014; Calahorro and Ruiz-Rubio 2011; Fernandez-Funez et al. 2015; Link 2005; Prussing et al. 2013). 
Table 2 Gene orthologs upregulated in both Aplysia SN aging and FL LOAD. All genes upregulated in two or more aging Aplysia SN differential expression data sets and five or more in meta-analysis of human frontal lobe Late Onset AD (FL LOAD) samples by Li et al. (2015). Aplysia RefSeq transcript identifiers, their BLAST-assigned putative human orthologs, and the e-value of the match are listed in the first three columns, with alternative names for each human gene in the fourth. The number of data sets in which these orthologs were upregulated is listed in columns 5 (Aplysia data sets) and $6(\mathrm{Li}$ et al. 2015 human FL LOAD data sets). Column 6 groups orthologs into broad categories relevant to aging and $\mathrm{AD}$ found in the discussion

\begin{tabular}{|c|c|c|c|c|c|c|}
\hline Aplysia RefSeq Transcript & e-value & Human gene symbol & Other names & $\begin{array}{l}\text { Aplysia } \\
\text { data } \\
\text { sets }\end{array}$ & $\begin{array}{l}\text { FL } \\
\text { LOAD } \\
\text { data sets }\end{array}$ & Major category \\
\hline XM_005091054 & $9.3 \mathrm{E}-70$ & ANKZ1 & ANKZF1, ZNF744 & 3 & 5 & Stress response (ER, ROS) \\
\hline XM_013084296 & $5.3 \mathrm{E}-09$ & BIRC3 & API2, MIHC, cIAP & 3 & 6 & Inflammation \\
\hline XM_013088003 & 7.2E-12 & BIRC3 & API2, MIHC, cIAP & 3 & 6 & Inflammation \\
\hline XM_005111747 & $5.3 \mathrm{E}-08$ & BIRC3 & API2, MIHC, cIAP & 2 & 6 & Inflammation \\
\hline XM_005102233 & $6.5 \mathrm{E}-22$ & BMP1 & mTID, PCP, TLD & 2 & 5 & $\begin{array}{l}\text { Inflammation, cholesterol } \\
\text { metabolism }\end{array}$ \\
\hline XM_005112068 & $4.2 \mathrm{E}-20$ & BTG1 & BTG1 & 2 & 6 & $\begin{array}{l}\text { Stress response (metabolic, } \\
\text { ER, ROS) }\end{array}$ \\
\hline XM_013080222 & $1.4 \mathrm{E}-86$ & CP3A5 & CYP3A5 & 2 & 5 & $\begin{array}{l}\text { Lipid metabolism, cholesterol } \\
\text { metabolism }\end{array}$ \\
\hline XM_005102749 & $1.1 \mathrm{E}-19$ & DDT4L & DDIT4L, REDD2 & 2 & 6 & Stress response (metabolic) \\
\hline XM_013089385 & $5.6 \mathrm{E}-17$ & GA45G & GADD45G, DDIT-2, CR6 & 3 & 5 & Stress response \\
\hline XM_005111489 & $3.2 \mathrm{E}-34$ & IKBA & NFKBIA, MAD3, NFKBI & 3 & 5 & Inflammation \\
\hline XM_013089050 & $4.9 \mathrm{E}-37$ & M3K8 & MAP3K8, COT, TPL2 & 2 & 5 & Inflammation \\
\hline XM_005095469 & 0 & MA2B1 & MAN2B1, LAMAN, MANB & 2 & 5 & Proteostasis \\
\hline NM_001204684 & $1.4 \mathrm{E}-135$ & MKNK2 & MNK2, GPRK7 & 2 & 6 & Inflammation \\
\hline XM_005108634 & $2.2 \mathrm{E}-25$ & MLXIP & MONDOA & 3 & 5 & Energy metabolism \\
\hline XM_005089580 & $6.6 \mathrm{E}-05$ & MUC1 & $\begin{array}{l}\text { CD227, PEM, EMA, EMA, } \\
\text { PEMT }\end{array}$ & 2 & 5 & $\begin{array}{l}\text { Stress response (ER), inflam- } \\
\text { mation }\end{array}$ \\
\hline XM_013081198 & $2.2 \mathrm{E}-15$ & MYD88 & MYD88 & 3 & 5 & Inflammation \\
\hline XM_005097661 & 4.4E-49 & NEO1 & NGN, IGDCC2 & 2 & 5 & $\begin{array}{l}\text { Iron accumulation, inflam- } \\
\text { mation }\end{array}$ \\
\hline XM_005108885 & 4.1E-21 & NFIL3 & E4BP, IL3BP1 & 2 & 5 & Inflammation \\
\hline XM_005096173 & $1.7 \mathrm{E}-12$ & NFKB1 & EBP1 & 2 & 5 & Inflammation \\
\hline XM_005091237 & $1.1 \mathrm{E}-77$ & SSRA & SSR1, TRAPA & 2 & 5 & Stress response (ER) \\
\hline XM_005110832 & $7.2 \mathrm{E}-43$ & TISB & $\begin{array}{l}\text { ZFP36L1, BRF1, ERF1, } \\
\text { TIS11B, BERG36, } \\
\text { RNF162B }\end{array}$ & 3 & 6 & $\begin{array}{l}\text { Inflammation, cholesterol } \\
\text { metabolism }\end{array}$ \\
\hline
\end{tabular}

Neurofibrillary tangles of hyperphosphorylated tau protein are also a hallmark of AD and several ADRDs. Tau neurofibrillary tangles do not naturally occur in invertebrate models; thus previous studies of tau hyperphosphorylation using Drosophila and C. elegans expressed altered human tau in invertebrate neurons to determine its detrimental effects (Alexander et al. 2014; Calahorro and Ruiz-Rubio 2011; Fernandez-Funez et al. 2015; Hannan et al. 2016; Link 2005; Moloney et al. 2010; Prussing et al. 2013; Sharma et al. 2017). These invertebrate models have been particularly useful in screening for the effects of taupathies in the nervous system (Hannan et al. 2016). Similarly, Aplysia SN do not naturally form tau neurofibrillary tangles; however, expression of mutant human tau also has been performed in Aplysia SN, which resulted in recapitulation of AD-like taupathies (Shemesh and Spira 2010, 2011). The presence of endogenous MAPT orthologs and the demonstrated capacity to induce taupathies in cultured neurons suggest that Aplysia SN may also offer an effective screening tool for the effects of hallmark AD proteinopathies on neurons.

The roughly 400 other orthologs of interest in Aplysia offer a broad landscape for functional investigation of the effects of amyloidopathies and taupathies on individual neurons and simple neural circuits. Given the success of translating molecular mechanisms of learning and memory from Aplysia to higher vertebrates and humans, the potential for investigation of AD mechanisms in Aplysia appears promising (Abrams 2012; Bailey et al. 1983; Ezzeddine and Glanzman 2003; Glanzman 2006; Kupfermann 1974; Lin and Glanzman 1994; Martin et al. 1997; Moroz 2011). This notion is further supported by the shared differential expression of 
Fig. 3 Orthologs in learning and memory pathway downregulated in common between Aplysia SN aging and FL LOAD. See Fig. 2 caption for diagram description. Commonly downregulated genes included major kinases of CREB1 (PKA, CAMK4, MEK1) and ELAV4, which stabilizes mRNAs of CREB1 target genes. This suggests that CREB1 signaling disruption is a common cause of cognitive impairment in Aplysia $\mathrm{SN}$ and LOAD

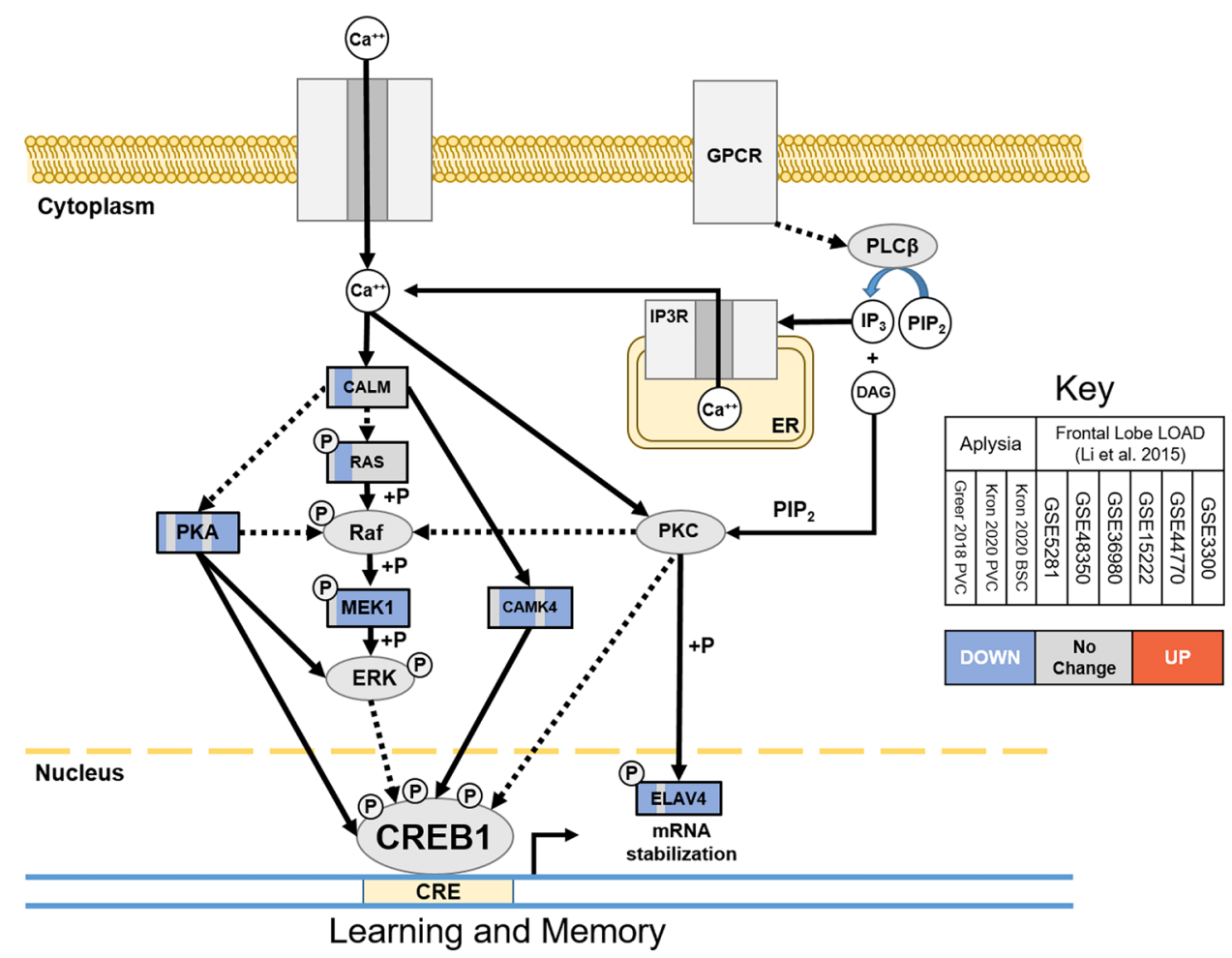

genes which are involved in processes known to play key roles in both neuronal aging and $\mathrm{AD}$, including learning and memory, neuronal signaling, transport of cellular cargo, energy metabolism, proteostasis, and neuroinflammation.

Memory impairment associated with AD has been suggested to be the result of synergistic toxicity between $\mathrm{A} \beta$ plaques and tau neurofibrillary tangles in cognitive centers like the frontal lobe and hippocampus. Gene transcription as a result of CREB activation is essential for memory formation across Metazoa (Silva et al. 1998). Disruption of CREB signaling in cognitive centers has been observed in AD brains as well as rodent and neuronal models of $\mathrm{AD}$ and is suggested to be a major component of AD-associated cognitive impairment (Puzzo et al. 2005; Snyder et al. 2005; Tong et al. 2001; Vitolo et al. 2002; Yamamoto-Sasaki et al. 1999). Similarly, Aplysia SN have been demonstrated to have impaired CREB signaling in aging (Greer et al. 2018; Kempsell and Fieber 2015a). As illustrated in Fig. 3, both aged Aplysia SN and human FL LOAD exhibited downregulation of orthologs of CAMKIV, MAP2K1, and PRKACA. These are critical components of the $\mathrm{Ca}^{++} /$calmodulin (Bito et al. 1996; Hardingham et al. 1998), MEK/ERK (Grewal et al. 2000; Li et al. 2019), and PKA (Turnham and Scott 2016) signaling cascades, respectively, that activate CREB during memory formation. Furthermore, commonly downregulated ELAV4 is a key effector of PKC that plays a critical role in stabilizing the mRNA of CREB target genes, facilitating protein translation and the establishment of CREB-dependent long-term memory in both species (Anderson et al. 2001; Deschenes-Furry et al. 2006; Mirisis et al. 2021; Pascale et al. 2004). Decreased activity and expression of these genes as a result of $A \beta$ and tau has been described previously in AD (Amadio et al. 2009; Gong et al. 2006; Hartmann et al. 2019; Vitolo et al. 2002; Yin et al. 2016b). This suggests that it is the dysregulation of key kinases and their effectors in the CREB signaling cascade that drives the cognitive impairments that typify both Aplysia SN aging and AD.

A mechanism by which $\mathrm{AD}$ is believed to impair cognitive function is via the disruption of normal vesicle dynamics and proper trafficking of cellular cargo (Barthet and Mulle 2020; Marsh and Alifragis 2018). Many of the putative orthologs downregulated in aging Aplysia SN and FL LOAD, namely NAPG (Inoue et al. 2015), ARF3 (Kondo et al. 2012), NECP1 (Ritter et al. 2003), and SNX4 (Traer et al. 2007), are involved in endosome formation and trafficking. Others, including NAPG (Stenbeck 1998), SYN2 (Cesca et al. 2010), SVOP (Janz et al. 1998), and EXOC8 (Guo et al. 1999), play key roles in vesicle docking and membrane fusion. Both SYN2 and NAPG have been shown to be disrupted in AD (Nie et al. 2017; Scheff and Price 2003; Sultana et al. 2006). This suggests that normal endo/exocytosis dynamics are affected in aging Aplysia SN as well as FL LOAD, possibly contributing to cognitive impairment. Transport of cellular cargo to and from the 
Table 3 Gene orthologs downregulated in both Aplysia SN aging and FL LOAD. All genes downregulated in two or more aging Aplysia SN differential expression data sets and five or more in meta-analysis of human frontal lobe FL LOAD samples by Li et al. (2015). See Table 2 for column descriptions. A majority of shared downregulated orthologs are involved in one or more of the following processes: cellular cargo transport, endo/exocytosis, proteostasis, lipid metabolism, energy metabolism, mitochondrial homeostasis, and signaling

\begin{tabular}{|c|c|c|c|c|c|c|}
\hline Aplysia RefSeq Transcript & e-value & Human gene symbol & Other names & $\begin{array}{l}\text { Aplysia } \\
\text { data } \\
\text { sets }\end{array}$ & $\begin{array}{l}\text { FL } \\
\text { LOAD } \\
\text { data sets }\end{array}$ & Major category \\
\hline XM_005098930 & 0 & AATM & GOT2 & 3 & 5 & Energy metabolism \\
\hline XM_005099066 & $2.5 \mathrm{E}-46$ & ARF3 & ARF3 & 2 & 6 & Cellular cargo transport \\
\hline XM_005112446 & $2.6 \mathrm{E}-25$ & CISD1 & ZCD1, mitoNEET & 2 & 5 & Energy metabolism \\
\hline XM_013080281 & $3.6 \mathrm{E}-21$ & CNRP1 & C2orf32 & 2 & 6 & Signaling \\
\hline XM_005098434 & $1.3 \mathrm{E}-59$ & CYC & CYCS & 2 & 6 & Energy metabolism \\
\hline XM_005096347 & $3.9 \mathrm{E}-65$ & DCTN6 & WS3 & 2 & 6 & Cellular cargo transport \\
\hline XM_005100966 & $1.1 \mathrm{E}-107$ & DECR2 & PDCR, SDR17C1 & 2 & 5 & Lipid metabolism \\
\hline XM_005092530 & $2.1 \mathrm{E}-146$ & ELAV4 & ELAVL4, HUD, PNEM & 2 & 6 & $\begin{array}{l}\text { Synaptic plasticity, mRNA } \\
\text { stabilization }\end{array}$ \\
\hline XM_005112819 & 0 & EXOC8 & EXO84 & 2 & 5 & Exocytosis \\
\hline XM_005097581 & $5.5 \mathrm{E}-10$ & FABPH & FABP3, FABP11, MDGI & 2 & 5 & Lipid metabolism \\
\hline XM_005096727 & $4.0 \mathrm{E}-41$ & GDAP1 & & 3 & 5 & Mitochondrial homeostasis \\
\hline XM_005111161 & $8.8 \mathrm{E}-44$ & GDAP1 & & 2 & 5 & Mitochondrial homeostasis \\
\hline NM_001204703 & 0 & GNAO & GNAO1 & 3 & 6 & Signaling, $\mathrm{Ca}^{++}$homeostasis \\
\hline XM_005102254 & 0 & GNB5 & & 2 & 5 & Signaling, $\mathrm{Ca}^{++}$homeostasis \\
\hline XM_005112007 & 0 & HMCS1 & HMGCS1,HMGCS & 2 & 5 & $\begin{array}{l}\text { Lipid metabolism, steroid } \\
\text { metabolism }\end{array}$ \\
\hline XM_005104774 & $4.8 \mathrm{E}-106$ & HPRT & HPRT1, HGPRT & 2 & 6 & Nucleotide salvage \\
\hline XM_005102830 & $2.0 \mathrm{E}-07$ & JUPI1 & ARM2, HN1 & 2 & 5 & Other \\
\hline NM_001204491 & 0 & KAPCA & PRKACA, PKACA & 2 & 5 & $\begin{array}{l}\text { Synaptic plasticity, } \mathrm{Ca}^{++} \\
\text {signaling, phosphorylation }\end{array}$ \\
\hline XM_005106951 & $4.0 \mathrm{E}-65$ & $\mathrm{KCC} 4$ & $\begin{array}{l}\text { CAMK4, CAMK, CAMK- } \\
\text { GR, CAMKIV }\end{array}$ & 2 & 5 & $\begin{array}{l}\text { Synaptic plasticity, } \mathrm{Ca}^{++} \\
\text {signaling, phosphorylation }\end{array}$ \\
\hline XM_005104905 & 0 & KIFA3 & KIFAP3, KIF3AP, SMAP & 3 & 6 & Cellular cargo transport \\
\hline XM_005102605 & $1.4 \mathrm{E}-10$ & LIAT1 & C17orf97 & 2 & 5 & Other \\
\hline XM_005098563 & 4.0E-171 & MDHC & MDH1, MDHA & 2 & 6 & Energy metabolism \\
\hline XM_005089329 & 0 & MP2K1 & $\begin{array}{l}\text { MAP2K1, MEK1, } \\
\text { PRKMK1, MKK1, } \\
\text { MAPKK1 }\end{array}$ & 2 & 6 & $\begin{array}{l}\text { Synaptic plasticity, phospho- } \\
\text { rylation }\end{array}$ \\
\hline XM_005098362 & $3.2 \mathrm{E}-56$ & MPND & MPND & 3 & 5 & Other \\
\hline XM_005089044 & 7.7E-36 & NDUAA & NDUFA10, CI-42kD & 2 & 5 & Energy metabolism \\
\hline XM_005097418 & 0 & NDUV1 & NDUFV1, UQOR1 & 2 & 5 & Energy metabolism \\
\hline XM_005099251 & $2.6 \mathrm{E}-103$ & NECP1 & NECAP1 & 2 & 6 & Endocytosis \\
\hline XM_005097828 & 0 & ODPB & PDHB, PHE1B & 3 & 6 & Energy metabolism \\
\hline XM_013084642 & $3.7 \mathrm{E}-89$ & OTUB1 & OTB1, OTU1 & 3 & 6 & DNA damage response \\
\hline XM_013081831 & 0 & PCCB & & 2 & 5 & Lipid metabolism \\
\hline XM_005089882 & 4.6E-28 & PEX19 & HK33, PXF & 2 & 5 & $\begin{array}{l}\text { Lipid metabolism, proteo- } \\
\text { stasis }\end{array}$ \\
\hline XM_005110189 & 0 & PFKAM & PFKM, PFKA, PFKX & 2 & 6 & Energy metabolism \\
\hline XM_005109909 & 4.9E-74 & PITH1 & PITHD1, C1orf128 & 2 & 5 & Transcription \\
\hline XM_005097948 & $2.5 \mathrm{E}-50$ & PPAC & ACP1, LMW-PTP & 2 & 6 & Phosphorylation \\
\hline XM_005097122 & $4.3 \mathrm{E}-133$ & RAB6A & RAB6 & 2 & 5 & Cellular cargo transport \\
\hline XM_005093164 & $1.7 \mathrm{E}-87$ & SAMC & SLC25A26 & 3 & 5 & Mitochondrial homeostasis \\
\hline XM_005108342 & $9.4 \mathrm{E}-28$ & $\mathrm{SCOC}$ & SCOCO & 3 & 6 & autophagy \\
\hline XM_005093202 & $4.4 \mathrm{E}-78$ & SNAG & NAPG, SNAPG & 2 & 6 & $\begin{array}{l}\text { Cellular cargo transport, } \\
\text { endocytosis }\end{array}$ \\
\hline
\end{tabular}


Table 3 (continued)

\begin{tabular}{|c|c|c|c|c|c|c|}
\hline Aplysia RefSeq Transcript & e-value & Human gene symbol & Other names & $\begin{array}{l}\text { Aplysia } \\
\text { data } \\
\text { sets }\end{array}$ & $\begin{array}{l}\text { FL } \\
\text { LOAD } \\
\text { data sets }\end{array}$ & Major category \\
\hline XM_013087712 & $1.4 \mathrm{E}-156$ & SNX4 & & 3 & 6 & $\begin{array}{l}\text { Cellular cargo transport, } \\
\text { endocytosis, proteostasis }\end{array}$ \\
\hline XM_005091494 & $2.5 \mathrm{E}-111$ & SPEE & $\begin{array}{l}\text { SRM, SPS1, SRML1, } \\
\text { SPDSY }\end{array}$ & 3 & 5 & $\begin{array}{l}\text { Mitochondrial homeostasis, } \\
\text { proteostasis }\end{array}$ \\
\hline NM_001204727 & $5.4 \mathrm{E}-129$ & STAU2 & STAU2 & 2 & 6 & Cellular cargo transport \\
\hline XM_013086901 & $5.0 \mathrm{E}-24$ & STUM & C1orf95 & 3 & 5 & Other \\
\hline XM_005107969 & 0 & SVOP & & 2 & 5 & Exocytosis \\
\hline NM_001204483 & $2.7 \mathrm{E}-151$ & SYN2 & & 2 & 5 & Exocytosis \\
\hline XM_013090258 & $1.1 \mathrm{E}-63$ & TTPAL & C20orf121 & 2 & 5 & Other \\
\hline XM_005091686 & $3.4 \mathrm{E}-21$ & TUSC2 & $\begin{array}{l}\text { C3orf11, FUS1, LGCC, } \\
\text { PDAP2 }\end{array}$ & 2 & 6 & $\begin{array}{l}\text { Mitochondrial homeostasis, } \\
\text { inflammation, cytokine } \\
\text { signaling, ROS response }\end{array}$ \\
\hline XM_005109532 & 8.7E-119 & $1433 Z$ & $\begin{array}{l}\text { YWHAZ, KCIP-1, 14-3-3 } \\
\text { protein zeta/delta }\end{array}$ & 2 & 5 & Signaling, proteostasis \\
\hline
\end{tabular}

synapse in response to synaptic activity is also central to synapse function and health (Guillaud et al. 2020; Hafezparast et al. 2003).

Both aging Aplysia SN and FL LOAD exhibit downregulation of DCTN6, a component of the dynein/dynactin complex that mediates retrograde transport, and RAB6A, the small GTPase that activates dynein-mediated transport (Yamada et al. 2013). This suggests common impairment of retrograde movement of cellular cargo. Similarly, common downregulation of KIFAP3, a key component of the kinesin motor, suggests that anterograde transport is impaired as well (Yamazaki et al. 1996). Furthermore, previously mentioned STAU2 and ELAVL4 both participate in kinesinmediated transport of mRNAs from the nucleus to neurites (Bronicki and Jasmin 2013; Tang et al. 2001). Anterograde transport of mitochondria and mRNA via kinesins is crucial for synapse health, learning, and memory, and disruptions of this process are associated with several neurodegenerative disorders (Guillaud et al. 2020). Disruption of mitochondrial transport in neurons also impairs mitochondrial homeostasis, which has been suggested to play a central role in many neurodegenerative disorders (Sheng and Cai 2012).

Mitochondrial dysfunction is a classic hallmark of neural aging and AD (Ferguson et al. 2005; Grimm and Eckert 2017; Ojaimi et al. 1999). Due to the energyintensive activity of neurons, any disruption in metabolic output can adversely affect signaling and synaptogenesis. The downregulation of several genes in common between Aplysia SN aging and FL LOAD suggest similar metabolic impairments. Downregulation of PKFM, the enzyme of the first committed step of glycolysis, but upregulation of glucose sensor and PFKM inducer MondoA, suggests common perturbation of glycolysis homeostasis (Sans et al. 2006). Furthermore, two components of the malate-aspartate shuttle (MAS), GOT2 and MDH1, are commonly downregulated. Disruption of MAS results in decoupling of cytosolic and mitochondrial NAD + /NADH ratios, which has been demonstrated to have adverse effects on mitochondrial metabolism and induce senescence (Bradshaw 2019; Broeks et al. 2019; Lautrup et al. 2019; $\mathrm{Xu}$ et al. 2020). Another common downregulated gene, PCCB, is critical for proper functioning of the mitochondrial tricarboxylic acid cycle (TCA) and has also been shown to be downregulated in a mouse model of AD (Franco et al. 2019). Dysfunction of PCC results in altered concentrations of TCA intermediates and accumulation of toxic metabolites, which decreases the activity of pyruvate dehydrogenase (PDH), the beta isoform of which is also downregulated (Wongkittichote et al. 2017). In addition to regulators of glycolysis and the TCA cycle, several components of mitochondrial oxidative phosphorylation are also commonly downregulated. These include components of mitochondrial respiratory complex I (NDUFA10, NDUFV1), cytochrome C (CYCS), which links complexes III and IV, and CISD1, which regulates maximal mitochondrial energy output (Kalpage et al. 2019; Paddock et al. 2007; Wang et al. 2017). These transcriptional signatures suggest similar impairment of mitochondrial energy metabolism in both Aplysia SN and FL LOAD. In addition to metabolic impairment, mitochondrial dysfunction also contributes to disrupted $\mathrm{Ca}^{++}$buffering in normal aging and AD (Pandya et al. 2015).

Proper mitochondrial $\mathrm{Ca}^{++}$regulation is critical not only for proper mitochondrial homeostatic functions but also for synaptic signaling (Gleichmann and Mattson 2011; Marchi et al. 2018; Satrustegui et al. 1996). In neurons, mitochondria act as critical sinks and reservoirs for $\mathrm{Ca}^{++}$ 
during signaling events. The signaling pathways that target CREB discussed earlier are themselves dependent upon tightly regulated $\mathrm{Ca}^{++}$signaling (Augustine et al. 2003). Impairment of mitochondrial $\mathrm{Ca}^{++}$homeostasis has been shown to contribute to $\mathrm{AD}$-associated proteinopathies and has even been suggested to be the proximal cause of $A D$ (Calvo-Rodriguez et al. 2020; Jadiya et al. 2019; Tong et al. 2018). Three genes downregulated in both aged Aplysia SN and FL LOAD, namely, GDAP1, TUSC2, and GN5B, play an important role in mitochondrial $\mathrm{Ca}^{++}$regulation, suggesting that aged Aplysia SN suffer similar disruptions of mitochondrial $\mathrm{Ca}^{++}$dynamics as human FL LOAD (Gonzalez-Sanchez et al. 2019; Kang et al. 2018; Uzhachenko et al. 2014, 2017). Mitochondrial impairment results in energy deprivation, generation of reactive oxygen species (ROS), and elevated $\mathrm{Ca}++$, which contribute to protein aggregation and associated endoplasmic reticulum (ER) stress. Sensors for these stressors converge in a single signaling process known as the integrated stress response (ISR) pathway.

Induction of the ISR results in decreased global translation via phosphorylation of eukaryotic initiation factor 2 (eIF2) and increased transcription of transcription factors in the activating transcription factor family, particularly ATF4 (Costa-Mattioli and Walter 2020; Pakos-Zebrucka et al. 2016). Increased proteostatic stress in $\mathrm{AD}$ due to $\mathrm{A} \beta$ plaques and tau neurofibrillary tangles has been demonstrated to increase eIF2 phosphorylation, suggesting increased ISR activity in AD (Chang et al. 2002; Ferrer 2002; Hernandez-Ortega et al. 2016; Hoozemans et al. 2005, 2009). Several putative orthologs upregulated in both aged Aplysia SN and FL LOAD are stress-induced genes, including DDIT4L (Cuaz-Perolin et al. 2004; Shoshani et al. 2002; Wang et al. 2003), BTG1 (Cho et al. 2003; Yuniati et al. 2019), SSR1 (Nagasawa et al. 2007), ANKZF1 (Tran et al. 2011; van Haaften-Visser et al. 2017), NFIL3 (Tamai et al. 2014), MUC1 (Olou et al. 2020), GAD45G (Liebermann and Hoffman 2008), and BIRC3 (Hamanaka et al. 2009; Warnakulasuriyarachchi et al. 2004). BTG1 enhances ISR signaling via interaction with ATF4 upon activation (Yuniati et al. 2016). Chronic induction of the ISR and resulting changes in the transcriptional and translational landscape of neurons has been suggested to play a role in disruptions of CREB-mediated learning and memory in $\mathrm{AD}$ (Hernandez-Ortega et al. 2016). NFIL3 has been shown to specifically inhibit CREB (MacGillavry et al. 2009). Similarly, upregulation of DDIT4L and NEO1 has been demonstrated to result in decreased neurogensis with impaired cognitive outcomes (Chen and Shifman 2019; Di Polo 2015; Metzger et al. 2007; Morquette et al. 2015; Shifman et al. 2009). Activation of the ISR also results in the secretion of cytokines that activate receptors in the toll-like and interleukin-like receptor (TIR) family (Abdel-Nour et al. 2019; Deng et al. 2004; Iwasaki et al. 2014). Activation of these TIR initiates signaling cascades that result in the translocation of transcription factors NF-kB and AP-1 to the nucleus and recruitment of pro-survival and proinflammatory genes.
Increased activation of proinflammatory signaling cascades recruited by the ISR has also been demonstrated to be increased in AD (Colangelo et al. 2002). Positive feedback of this proinflammatory loop has been proposed to induce chronic neuroinflammation and contribute to neurodegenerative consequences in AD (Jones and Kounatidis 2017; Ju Hwang et al. 2019; Lindsay et al. 2021; Uddin et al. 2021). For example, induction of miRNAs by NF- $\kappa \mathrm{B}$ in AD directly results in the downregulation of previously discussed SYN2 (Lukiw 2012). Several genes that participate in and are recruited by the signaling cascades downstream of TIR are upregulated in both Aplysia SN aging and human FL LOAD (Fig. 2), including MYD88, MAP3K8 (Chorzalska et al. 2017), and MKNK2 (Bao et al. 2017; Xu et al. 2018). Furthermore, NEO1discussed previously exhibits strong proinflammatory effects (Chen and Shifman 2019; Fujita and Yamashita 2017; Shifman et al. 2009). Most significantly, many core components of the quintessential proinflammatory signaling cascade, NF-kB signaling, are commonly upregulated. NF-KB1, also known as p105, is an NF-kB family protein that, upon phosphorylation as a result of MYD88 activation, is degraded by the proteosome. This liberates MAP3K8, which initiates the AP-1 branch of proinflammatory signaling and produces the $\mathrm{p} 50 \mathrm{NF}-\mathrm{kB}$ subunit, which is then recruited into homodimers or heterodimers with $\mathrm{p} 65$ to activate downstream NF-kB target genes (Beinke et al. 2004). Several of these target genes are commonly upregulated, including NFKBIA (Hay et al. 1999; Sun et al. 1993), BCL3 (Bours et al. 1993; Caamano et al. 1996; Edwards et al. 2015; Saito et al. 2010), and BIRC3 (Hu et al. 2004; James et al. 2006; Simon et al. 2007). Common upregulation of key genes in this pathway suggest that increased proinflammatory signaling as a result of increased cellular stress is a relevant component of Aplysia SN aging and FL LOAD. However, few of these relationships have been experimentally validated in Aplysia.

While these genes have been observed to play key roles in human neurodegenerative disease, orthologs of these genes have been demonstrated to have conserved function and stressassociated upregulation and function in invertebrate models. Molluscan orthologs of BTG1 (Peng et al. 2014), NFIL3 (Li et al. 2017), MYD88 (Zhang et al. 2015), and BIRC3 (Wang et al. 2016) have been demonstrated to be activated by biotic and abiotic stressors in bivalves. Several other dysregulated orthologs, including NAPG (Clary et al. 1990), SNX4 (Nemec et al. 2017), EXOC8 (Guo et al. 1999), ANKZF1 (Tran et al. 2011), and DDIT4L (Reiling and Hafen 2004) have conserved function between humans and models considered more divergent from humans than Aplysia (Moroz et al. 2006), including ecdysozoans like Drosophila and C. elegans and even yeast. Thus, we believe it plausible that dysregulation of these genes will have similar outcomes in Aplysia SN as observed in human neurons.

Differential expression of genes shared between Aplysia $\mathrm{SN}$ aging and FL LOAD represents critical pathways that are 
disrupted in aging and neurodegenerative disease, including mitochondrial homeostasis, energy metabolism, vesicle dynamics, cellular cargo transport, $\mathrm{Ca}^{++}$homeostasis, and synaptic plasticity (Di Paolo and Kim 2011; Haas 2019; Jang et al. 2018; Lopez-Otin et al. 2013; Martinez et al. 2017; Wong et al. 2020; Wu et al. 2019; Yin et al. 2016a). Although the hallmark pathologies of $\mathrm{AD}$ are only known in humans, these data suggest that, while the proximal source of neuronal stress may be different, similar transcriptional changes as a result of cellular stress underpin cognitive impairment in both Aplysia SN aging and AD. Indeed, the commonalities between aging Aplysia SN and FL LOAD expression patterns make sense in light of the current understanding that normal brain aging and dementias like $\mathrm{AD}$ are parts of a continuum of neurodegenerative outcomes associated with aging (Franceschi et al. 2018). While surface receptors and downstream effectors have diverged and specialized differently over the course of evolution, these data suggest that orthologous signaling cascades and their disruption as a result of age-associated stressors are conserved between the human frontal lobe and Aplysia sensory neurons. We strongly believe that these results, in addition to previous studies, demonstrate the excellent applicability of Aplysia as a multivalent model for the study of AD and ADRD.

Supplementary Information The online version contains supplementary material available at https://doi.org/10.1007/s12031-021-01918-3.

Acknowledgements We gratefully acknowledge the University of Miami Aplysia Resource staff for their assistance. We also thank Dr. Justin Greer for his valuable insight and advice.

Authors' Contributions Both authors designed this study. Nicholas S. Kron collected and analysed the data. Nicholas S. Kron wrote the manuscript with input from Lynne A. Fieber. Both authors read and approved the final manuscript.

Funding This work was funded by the National Institutes of Health Grant (P40OD010952). The funders had no role in study design, data collection and analysis, decision to publish, or preparation of the manuscript.

Availability of Data and Materials Data used in this study is freely available from the cited publications and public databases from which it was sourced as described in the text.

Code Availability Code used for this study is available at the following GitHub repository: [https://github.com/Nicholas-Kron/Kron_Aplysia_ Alzheimer-s_Model].

\section{Declarations}

Ethics Approval and Consent to Participate Not applicable

\section{Consent for Publication Not applicable}

Competing Interests The authors declare that the research was conducted in the absence of any commercial or financial relationships that could be construed as a potential conflict of interest.
Open Access This article is licensed under a Creative Commons Attribution 4.0 International License, which permits use, sharing, adaptation, distribution and reproduction in any medium or format, as long as you give appropriate credit to the original author(s) and the source, provide a link to the Creative Commons licence, and indicate if changes were made. The images or other third party material in this article are included in the article's Creative Commons licence, unless indicated otherwise in a credit line to the material. If material is not included in the article's Creative Commons licence and your intended use is not permitted by statutory regulation or exceeds the permitted use, you will need to obtain permission directly from the copyright holder. To view a copy of this licence, visit http://creativecommons.org/licenses/by/4.0/.

\section{References}

Abdel-Nour M et al (2019) The heme-regulated inhibitor is a cytosolic sensor of protein misfolding that controls innate immune signaling. Science 365

Abrams TW (2012) Studies on Aplysia neurons suggest treatments for chronic human disorders. Curr Biol 22:R705-R711

Alexander AG et al (2014) Use of Caenorhabditis elegans as a model to study Alzheimer's disease and other neurodegenerative diseases. Front Genet 5:279

Amadio M et al (2009) nELAV proteins alteration in Alzheimer's disease brain: a novel putative target for amyloid-beta reverberating on AbetaPP processing. J Alzheimers Dis 16:409-419

Anderson KD et al (2001) Overexpression of HuD accelerates neurite outgrowth and increases GAP-43 mRNA expression in cortical neurons and retinoic acid-induced embryonic stem cells in vitro. Exp Neurol 168:250-258

Augustine GJ et al (2003) Local calcium signaling in neurons. Neuron 40:331-346

Bailey CH et al (1983) Behavioral changes in aging Aplysia: a model system for studying the cellular basis of age-impaired learning, memory, and arousal. Behav Neural Biol 38:70-81

Bao Y et al (2017) Brd4 modulates the innate immune response through Mnk2-eIF4E pathway-dependent translational control of IkappaBalpha. Proc Natl Acad Sci U S A 114:E3993-E4001

Barthet G, Mulle C (2020) Presynaptic failure in Alzheimer's disease. Prog Neurobiol 194:101801

Baxter DA, Byrne JH (2006) Feeding behavior of Aplysia: a model system for comparing cellular mechanisms of classical and operant conditioning. Learn Mem 13:669-680

Beinke S et al (2004) Lipopolysaccharide activation of the TPL-2/ MEK/extracellular signal-regulated kinase mitogen-activated protein kinase cascade is regulated by IkappaB kinase-induced proteolysis of NF-kappaB1 p105. Mol Cell Biol 24:9658-9667

Bertram L et al (2007) Systematic meta-analyses of Alzheimer disease genetic association studies: the AlzGene database. Nat Genet 39:17-23

Bito $\mathrm{H}$ et al (1996) CREB phosphorylation and dephosphorylation: a $\mathrm{Ca}(2+)$ - and stimulus duration-dependent switch for hippocampal gene expression. Cell 87:1203-1214

Bours V et al (1993) The oncoprotein Bcl-3 directly transactivates through kappa B motifs via association with DNA-binding p50B homodimers. Cell 72:729-739

Bradshaw PC (2019) Cytoplasmic and Mitochondrial NADPH-Coupled Redox Systems in the Regulation of Aging. Nutrients 11:504

Broeks MH et al (2019) MDH1 deficiency is a metabolic disorder of the malate-aspartate shuttle associated with early onset severe encephalopathy. Hum Genet 138:1247-1257

Bronicki LM, Jasmin BJ (2013) Emerging complexity of the HuD/ ELAV14 gene; implications for neuronal development, function, and dysfunction. RNA 19:1019-1037 
Caamano JH et al (1996) Constitutive expression of Bc1-3 in thymocytes increases the DNA binding of NF-kappaB1 (p50) homodimers in vivo. Mol Cell Biol 16:1342-1348

Calahorro F, Ruiz-Rubio M (2011) Caenorhabditis elegans as an experimental tool for the study of complex neurological diseases: Parkinson's disease, Alzheimer's disease and autism spectrum disorder. Invert Neurosci 11:73-83

Calvo-Rodriguez M et al (2020) Increased mitochondrial calcium levels associated with neuronal death in a mouse model of Alzheimer's disease. Nat Commun 11:2146

Camacho C et al (2009) BLAST plus : architecture and applications. Bmc Bioinformatics 10

Carew TJ et al (1983) Differential classical conditioning of a defensive withdrawal reflex in Aplysia californica. Science 219:397-400

Carlson M (2019) org.Hs.eg.db: Genome wide annotation for Human. Bioconductor

Castellucci V et al (1970) Neuronal mechanisms of habituation and dishabituation of the gill-withdrawal reflex in Aplysia. Science 167:1745-1748

Cesca F et al (2010) The synapsins: key actors of synapse function and plasticity. Prog Neurobiol 91:313-348

Chang RCC et al (2002) Phosphorylation of eukaryotic initiation factor- $2 a($ IF $2 a)$ is associated with neuronal degeneration in Alzheimer's disease. NeuroReport 13:2429-2432

Chen J, Shifman MI (2019) Inhibition of neogenin promotes neuronal survival and improved behavior recovery after spinal cord injury. Neuroscience 408:430-447

Cho 1 et al (2003) Btg-1 Induction by Oxidative Stres. 춘계총회 및 학 술 대회. 151-151

Chorzalska AD et al (2017) Map3k8/COT1/Tpl2 Drives Resistance to Bcr-Abl1 Inhibition through Activation of MEK-ERK and NF-Kb Pathways. Blood 130:2529-2529

Clary DO et al (1990) SNAPs, a family of NSF attachment proteins involved in intracellular membrane fusion in animals and yeast. Cell 61:709-721

Cleary LJ et al (1998) Cellular correlates of long-term sensitization in Aplysia. J Neurosci 18:5988-5998

Colangelo V et al (2002) Gene expression profiling of 12633 genes in Alzheimer hippocampal CA1: transcription and neurotrophic factor down-regulation and up-regulation of apoptotic and proinflammatory signaling. J Neurosci Res 70:462-473

Costa-Mattioli M, Walter P (2020) The integrated stress response: From mechanism to disease. Science 368

Crous-Bou M et al (2017) Alzheimer's disease prevention: from risk factors to early intervention. Alzheimers Res Ther 9:71

Cuaz-Perolin C et al (2004) REDD2 gene is upregulated by modified LDL or hypoxia and mediates human macrophage cell death. Arterioscler Thromb Vasc Biol 24:1830-1835

Deng J et al (2004) Translational repression mediates activation of nuclear factor kappa B by phosphorylated translation initiation factor 2. Mol Cell Biol 24:10161-10168

Deschenes-Furry J et al (2006) The RNA-binding protein HuD: a regulator of neuronal differentiation, maintenance and plasticity. BioEssays 28:822-833

Di Paolo G, Kim TW (2011) Linking lipids to Alzheimer's disease: cholesterol and beyond. Nat Rev Neurosci 12:284-296

Di Polo A (2015) Dendrite pathology and neurodegeneration: focus on mTOR. Neural Regen Res 10:559-561

Edwards RH et al (2015) Changes in expression induced by EpsteinBarr Virus LMP1-CTAR1: potential role of bcl3. mBio 6

Ezzeddine Y, Glanzman DL (2003) Prolonged habituation of the gillwithdrawal reflex in Aplysia depends on protein synthesis, protein phosphatase activity, and postsynaptic glutamate receptors. J Neurosci 23:9585-9594
Ferguson M et al (2005) Age-associated decline in mitochondrial respiration and electron transport in Drosophila melanogaster. Biochemical Journal 390:501-511

Fernandez-Funez P et al (2015) Modeling the complex pathology of Alzheimer's disease in Drosophila. Exp Neurol 274:58-71

Ferrer I (2002) Differential expression of phosphorylated translation initiation factor 2 alpha in Alzheimer's disease and CreutzfeldtJakob's disease. Neuropathol Appl Neurobiol 28:441-451

Franceschi C et al (2018) The Continuum of Aging and Age-Related Diseases: Common Mechanisms but Different Rates. Front Med (lausanne) 5:61

Franco R et al (2019) Lessons on Differential Neuronal-DeathVulnerability from Familial Cases of Parkinson's and Alzheimer's Diseases. Int J Mol Sci 20

Fujita Y, Yamashita T (2017) The roles of RGMa-neogenin signaling in inflammation and angiogenesis. Inflamm Regen 37:6

Glanzman DL (2006) The cellular mechanisms of learning in Aplysia: Of blind men and elephants. Biol Bull 210:271-279

Gleichmann M, Mattson MP (2011) Neuronal calcium homeostasis and dysregulation. Antioxid Redox Signal 14:1261-1273

Gong B et al (2006) Ubiquitin hydrolase Uch-L1 rescues betaamyloid-induced decreases in synaptic function and contextual memory. Cell 126:775-788

Gonzalez-Sanchez P et al (2019) Calcium Deregulation and Mitochondrial Bioenergetics in GDAP1-Related CMT Disease. Int J Mol Sci 20

Gotz J, Ittner LM (2008) Animal models of Alzheimer's disease and frontotemporal dementia. Nat Rev Neurosci 9:532-544

Greer JB et al (2019) Altered expression of ionotropic L-Glutamate receptors in aged sensory neurons of Aplysia californica. PloS One 14

Greer JB et al (2018) Whole-transcriptome changes in gene expression accompany aging of sensory neurons in Aplysia californica. BMC Genomics 19:529

Grewal SS et al (2000) Calcium and cAMP signals differentially regulate cAMP-responsive element-binding protein function via a Rap1-extracellular signal-regulated kinase pathway. J Biol Chem 275:34433-34441

Grimm A, Eckert A (2017) Brain aging and neurodegeneration: from a mitochondrial point of view. J Neurochem 143:418-431

Guillaud L et al (2020) Anterograde Axonal Transport in Neuronal Homeostasis and Disease. Front Mol Neurosci 13:556175

Guo W et al (1999) Exo84p is an exocyst protein essential for secretion. J Biol Chem 274:23558-23564

Haas RH (2019) Mitochondrial Dysfunction in Aging and Diseases of Aging. Biology (Basel) 8

Hafezparast M et al (2003) Mutations in dynein link motor neuron degeneration to defects in retrograde transport. Science 300:808-812

Hallahan BJ et al (1992) Increased age affects properties characterizing behavioral plasticity in freely behaving Aplysia. Neurobiol Aging 13:217-225

Hamanaka RB et al (2009) PERK-dependent regulation of IAP translation during ER stress. Oncogene 28:910-920

Hannan SB et al (2016) Cellular and molecular modifier pathways in tauopathies: the big picture from screening invertebrate models. J Neurochem 137:12-25

Hardingham GE et al (1998) Mechanisms controlling gene expression by nuclear calcium signals. Cell Calcium 23:131-134

Hartmann A et al (2019) Influence of RNA-binding proteins on Alzheimer's disease and related cognitive endophenotypes. Pharmacopsychiatry 52(P3):2

Hay RT et al (1999) Control of NF-kappa B transcriptional activation by signal induced proteolysis of I kappa B alpha. Philos Trans R Soc Lond B Biol Sci 354:1601-1609 
Hernandez-Ortega K et al (2016) Altered Machinery of Protein Synthesis in Alzheimer's: From the Nucleolus to the Ribosome. Brain Pathol 26:593-605

Hoozemans JJ et al (2005) The unfolded protein response is activated in Alzheimer's disease. Acta Neuropathol 110:165-172

Hoozemans JJ et al (2009) The unfolded protein response is activated in pretangle neurons in Alzheimer's disease hippocampus. Am J Pathol 174:1241-1251

$\mathrm{Hu} \mathrm{P}$ et al (2004) Critical role of endogenous Akt/IAPs and MEK1/ ERK pathways in counteracting endoplasmic reticulum stressinduced cell death. J Biol Chem 279:49420-49429

Hu YS et al (2017) Analyzing the genes related to Alzheimer's disease via a network and pathway-based approach. Alzheimers Res Ther 9:29

Inoue $\mathrm{H}$ et al (2015) $\gamma$-SNAP stimulates disassembly of endosomal SNARE complexes and regulates endocytic trafficking pathways. J Cell Sci 128:2781-2794

Iwasaki $\mathrm{Y}$ et al (2014) Activating transcription factor 4 links metabolic stress to interleukin-6 expression in macrophages. Diabetes 63:152-161

Jack CR Jr et al (2018) NIA-AA Research Framework: Toward a biological definition of Alzheimer's disease. Alzheimers Dement $14: 535-562$

Jadiya $P$ et al (2019) Impaired mitochondrial calcium efflux contributes to disease progression in models of Alzheimer's disease. Nat Commun 10:3885

James MA et al (2006) Human papillomavirus type 16 E6 activates NF-kappaB, induces cIAP-2 expression, and protects against apoptosis in a PDZ binding motif-dependent manner. J Virol 80:5301-5307

Jang DH et al (2005) Cofilin expression induces cofilin-actin rod formation and disrupts synaptic structure and function in Aplysia synapses. Proc Natl Acad Sci U S A 102:16072-16077

Jang JY et al (2018) The role of mitochondria in aging. J Clin Invest 128:3662-3670

Janz R et al (1998) SVOP, an Evolutionarily Conserved Synaptic Vesicle Protein, Suggests Novel Transport Functions of Synaptic Vesicles. J Neurosci 18:9269-9281

Jones SV, Kounatidis I (2017) Nuclear Factor-Kappa B and Alzheimer Disease, Unifying Genetic and Environmental Risk Factors from Cell to Humans. Front Immunol 8:1805

Ju Hwang C et al (2019) NF-kappaB as a Key Mediator of Brain Inflammation in Alzheimer's Disease. CNS Neurol Disord Drug Targets 18:3-10

Kadakkuzha BM et al (2013) Age-associated bidirectional modulation of gene expression in single identified R15 neuron of Aplysia. BMC Genomics 14:880

Kalpage HA et al (2019) Tissue-specific regulation of cytochrome $\mathrm{c}$ by posttranslational modifications: respiration, the mitochondrial membrane potential, ROS, and apoptosis. FASEB J 33:1540-1553

Kang $\mathrm{N}$ et al (2018) Increased store-operated $\mathrm{Ca}(2+)$ entry mediated by GNB5 and STIM1. Korean J Physiol Pharmacol 22:343-348

Kempsell AT, Fieber LA (2014) Behavioral aging is associated with reduced sensory neuron excitability in Aplysia californica. Front Aging Neurosci 6

Kempsell AT, Fieber LA (2015a) Age-related deficits in synaptic plasticity rescued by activating PKA or PKC in sensory neurons of Aplysia californica. Front Aging Neurosci 7

Kempsell AT, Fieber LA (2015b) Aging in sensory and motor neurons results in learning failure in Aplysia californica. PloS One 10

Kempsell AT, Fieber LA (2016) Habituation in the tail withdrawal reflex circuit is impaired during aging in Aplysia californica. Front Aging Neurosci 8

Kindy MS et al (1991) Age-related differential expression of neuropeptide mRNAs in Aplysia. NeuroReport 2:465-468
Klein M et al (1982) Serotonin modulates a specific potassium current in the sensory neurons that show presynaptic facilitation in Aplysia. Proc Natl Acad Sci U S A 79:5713-5717

Kondo Y et al (2012) ARF1 and ARF3 are required for the integrity of recycling endosomes and the recycling pathway. Cell Struct Funct 37:141-154

Kron NS et al (2020) Changes in metabolism and proteostasis drive aging phenotype in Aplysia californica sensory neurons. Front Aging Neurosci 12

Kupfermann I (1974) Feeding behavior in Aplysia: a simple system for the study of motivation. Behav Biol 10:1-26

Lautrup S et al (2019) NAD(+) in Brain Aging and Neurodegenerative Disorders. Cell Metab 30:630-655

$\mathrm{Li} J$ et al (2019) The MEK/ERK/CREB signaling pathway is involved in atrazine induced hippocampal neurotoxicity in Sprague Dawley rats. Ecotoxicol Environ Saf 170:673-681

Li J et al (2017) The first invertebrate NFIL3 transcription factor with role in immune defense identified from the Hong Kong oyster. Crassostrea Hongkongensis Dev Comp Immunol 76:1-8

Li X et al (2015) Integrated genomic approaches identify major pathways and upstream regulators in late onset Alzheimer's disease. Sci Rep 5:12393

Liebermann DA, Hoffman B (2008) Gadd45 in stress signaling. J Mol Signal 3:15

Lin XY, Glanzman DL (1994) Hebbian induction of long-term potentiation of Aplysia sensorimotor synapses: partial requirement for activation of an NMDA-related receptor. Proc Biol Sci 255:215-221

Lindsay A et al (2021) A nuclear factor-kappa B inhibiting peptide suppresses innate immune receptors and gliosis in a transgenic mouse model of Alzheimer's disease. Biomed Pharmacother $138: 111405$

Link CD (2005) Invertebrate models of Alzheimer's disease. Genes Brain Behav 4:147-156

Lopez-Otin C et al (2013) The hallmarks of aging. Cell 153:1194-1217

Lukiw WJ (2012) NF-kappaB-regulated, proinflammatory miRNAs in Alzheimer's disease. Alzheimers Res Ther 4:47

MacGillavry HD et al (2009) NFIL3 and cAMP response elementbinding protein form a transcriptional feedforward loop that controls neuronal regeneration-associated gene expression. J Neurosci 29:15542-15550

Marchi S et al (2018) Mitochondrial and endoplasmic reticulum calcium homeostasis and cell death. Cell Calcium 69:62-72

Marsh J, Alifragis P (2018) Synaptic dysfunction in Alzheimer's disease: the effects of amyloid-beta on synaptic vesicle dynamics as a novel target for therapeutic intervention. Neural Regen Res 13:616-623

Martin KC et al (1997) MAP kinase translocates into the nucleus of the presynaptic cell and is required for long-term facilitation in Aplysia. Neuron 18:899-912

Martinez G et al (2017) Endoplasmic reticulum proteostasis impairment in aging. Aging Cell 16:615-623

Medina M, Avila J (2014) The need for better AD animal models. Front Pharmacol 5:227

Metzger M et al (2007) RGMa inhibits neurite outgrowth of neuronal progenitors from murine enteric nervous system via the neogenin receptor in vitro. J Neurochem 103:2665-2678

Mirisis AA et al (2021) ELAV Proteins Bind and Stabilize C/EBP mRNA in the Induction of Long-Term Memory in Aplysia. J Neurosci 41:947-959

Moloney A et al (2010) Alzheimer's disease: insights from Drosophila melanogaster models. Trends Biochem Sci 35:228-235

Moroz LL (2011) Aplysia. Curr Biol 21:R60-R61

Moroz LL et al (2006) Neuronal transcriptome of Aplysia: neuronal compartments and circuitry. Cell 127:1453-1467 
Moroz LL, Kohn AB (2010) Do different neurons age differently? Direct genome-wide analysis of aging in single identified cholinergic neurons. Front Aging Neurosci 2

Moroz LL, Kohn AB (2013) Single-neuron transcriptome and methylome sequencing for epigenomic analysis of aging. Methods Mol Biol 1048:323-352

Morquette B et al (2015) REDD2-mediated inhibition of mTOR promotes dendrite retraction induced by axonal injury. Cell Death Differ 22:612-625

Nagasawa K et al (2007) Simultaneous induction of the four subunits of the TRAP complex by ER stress accelerates ER degradation. EMBO Rep 8:483-489

Nemec AA et al (2017) Autophagic clearance of proteasomes in yeast requires the conserved sorting nexin Snx4. J Biol Chem 292:21466-21480

Nie L et al (2017) Ginsenoside Rg1 Ameliorates Behavioral Abnormalities and Modulates the Hippocampal Proteomic Change in Triple Transgenic Mice of Alzheimer's Disease. Oxid Med Cell Longev 2017:6473506

Ojaimi J et al (1999) Mitochondrial respiratory chain activity in the human brain as a function of age. Mech Ageing Dev 111:39-47

Olou AA et al (2020) MUC1 oncoprotein mitigates ER stress via CDAmediated reprogramming of pyrimidine metabolism. Oncogene 39:3381-3395

Paddock ML et al (2007) MitoNEET is a uniquely folded 2Fe 2S outer mitochondrial membrane protein stabilized by pioglitazone. Proc Natl Acad Sci U S A 104:14342-14347

Pakos-Zebrucka K et al (2016) The integrated stress response. EMBO Rep 17:1374-1395

Pandya JD et al (2015) Decreased mitochondrial bioenergetics and calcium buffering capacity in the basal ganglia correlates with motor deficits in a nonhuman primate model of aging. Neurobiol Aging 36:1903-1913

Papka R et al (1981) Age-Dependent Anatomical Changes in an Identified Neuron in the Cns of Aplysia-Californica. J Neurobiol 12:455-468

Pascale A et al (2004) Increase of the RNA-binding protein HuD and posttranscriptional up-regulation of the GAP-43 gene during spatial memory. Proc Natl Acad Sci U S A 101:1217-1222

Peng K et al (2014) Molecular cloning, sequence analysis, and cadmium stress-rated expression changes of BTG1 in freshwater pearl mussel (Hyriopsis schlegelii). Dongwuxue Yanjiu 35:389-397

Peretz B et al (1984) Functional History of Two Motor Neurons and the Morphometry of Their Neuromuscular Junctions in the Gill of Aplysia: Evidence for Differential Aging PNAS 81:4232-4236

Peron R et al (2018) Alpha-Secretase ADAM10 Regulation: Insights into Alzheimer's Disease Treatment. Pharmaceuticals (Basel) 11

Prussing K et al (2013) Drosophila melanogaster as a model organism for Alzheimer's disease. Mol Neurodegener 8:35

Puzzo D et al (2005) Amyloid-beta peptide inhibits activation of the nitric oxide/cGMP/cAMP-responsive element-binding protein pathway during hippocampal synaptic plasticity. J Neurosci 25:6887-6897

R Core Team (2013) R: A language and environment for statistical computing. R Foundation for Statistical Computing, Vienna, Austria

Rattan KS, Peretz B (1981) Age-dependent behavioral changes and physiological changes in identified neurons in Aplysia californica. J Neurobiol 12:469-478

Reiling JH, Hafen E (2004) The hypoxia-induced paralogs Scylla and Charybdis inhibit growth by down-regulating S6K activity upstream of TSC in Drosophila. Genes Dev 18:2879-2892

Ritter B et al (2003) Identification of a family of endocytic proteins that define a new alpha-adaptin ear-binding motif. EMBO Rep 4:1089-1095
Saito K et al (2010) Activation of the PI3K-Akt pathway by human T cell leukemia virus type 1 (HTLV-1) oncoprotein Tax increases $\mathrm{Bcl} 3$ expression, which is associated with enhanced growth of HTLV-1-infected T cells. Virology 403:173-180

Sans CL et al (2006) MondoA-Mlx heterodimers are candidate sensors of cellular energy status: Mitochondrial localization and direct regulation of glycolysis. Mol Cell Biol 26:4863-4871

Satrustegui J et al (1996) Cytosolic and mitochondrial calcium in synaptosomes during aging. Life Sci 59:429-434

Sawada M, Ichinose M (1996) Amyloid-beta proteins reduce the GABA-induced Cl- current in identified Aplysia neurons. Neurosci Lett 213:213-215

Scheff SW, Price DA (2003) Synaptic pathology in Alzheimer's disease: a review of ultrastructural studies. Neurobiol Aging 24:1029-1046

Schneider LS et al (2014) Clinical trials and late-stage drug development for Alzheimer's disease: an appraisal from 1984 to 2014. J Intern Med 275:251-283

Sharma N et al (2017) Lower vertebrate and invertebrate models of Alzheimer's disease - A review. Eur J Pharmacol 815:312-323

Shemesh OA, Spira ME (2010) Hallmark cellular pathology of Alzheimer's disease induced by mutant human tau expression in cultured Aplysia neurons. Acta Neuropathol 120:209-222

Shemesh OA, Spira ME (2011) Rescue of neurons from undergoing hallmark tau-induced Alzheimer's disease cell pathologies by the antimitotic drug paclitaxel. Neurobiol Dis 43:163-175

Sheng ZH, Cai Q (2012) Mitochondrial transport in neurons: impact on synaptic homeostasis and neurodegeneration. Nat Rev Neurosci 13:77-93

Shifman MI et al (2009) Expression of the repulsive guidance molecule RGM and its receptor neogenin after spinal cord injury in sea lamprey. Exp Neurol 217:242-251

Shoshani T et al (2002) Identification of a novel hypoxia-inducible factor 1-responsive gene, RTP801, involved in apoptosis. Mol Cell Biol 22:2283-2293

Silva AJ et al (1998) CREB and memory. Annu Rev Neurosci 21:127-148

Simon P et al (2007) Inhibitor of apoptosis protein BIRC3 (API2, cIAP2, AIP1) is upregulated by the non-canonical NFKB pathway. Can Res 67:5327-5327

Snyder EM et al (2005) Regulation of NMDA receptor trafficking by amyloid-beta. Nat Neurosci 8:1051-1058

Srivatsan M, Peretz B (1996) Effect of acetylcholinesterase inhibition on behavior is age-dependent in freely moving Aplysia. Behav Brain Res 77:115-124

Stenbeck G (1998) Soluble NSF-attachment proteins. Int J Biochem Cell Biol 30:573-577

Sultana R et al (2006) Redox proteomics identification of oxidized proteins in Alzheimer's disease hippocampus and cerebellum: an approach to understand pathological and biochemical alterations in AD. Neurobiol Aging 27:1564-1576

Sun SC et al (1993) NF-kappa B controls expression of inhibitor I kappa B alpha: evidence for an inducible autoregulatory pathway. Science 259:1912-1915

Surguchov A (2021) Invertebrate Models Untangle the Mechanism of Neurodegeneration in Parkinson's Disease. Cells 10:407

Tamai S et al (2014) Neuroprotective role of the basic leucine zipper transcription factor NFIL3 in models of amyotrophic lateral sclerosis. J Biol Chem 289:1629-1638

Tang SJ et al (2001) A role for a rat homolog of staufen in the transport of RNA to neuronal dendrites. Neuron 32:463-475

Tong BC et al (2018) Calcium signaling in Alzheimer's disease \& therapies. Biochim Biophys Acta Mol Cell Res 1865:1745-1760

Tong L et al (2001) Beta -amyloid-(1-42) impairs activity-dependent cAMP-response element-binding protein signaling in neurons 
at concentrations in which cell survival Is not compromised. $\mathbf{J}$ Biol Chem 276:17301-17306

Traer CJ et al (2007) SNX4 coordinates endosomal sorting of TfnR with dynein-mediated transport into the endocytic recycling compartment. Nat Cell Biol 9:1370-1380

Tran JR et al (2011) A Cdc48p-associated factor modulates endoplasmic reticulum-associated degradation, cell stress, and ubiquitinated protein homeostasis. J Biol Chem 286:5744-5755

Turnham RE, Scott JD (2016) Protein kinase A catalytic subunit isoform PRKACA; History, function and physiology. Gene 577:101-108

Uddin MS et al (2021) Anti-Neuroinflammatory Potential of Polyphenols by Inhibiting NF-kappaB to Halt Alzheimer's Disease. Curr Pharm Des 27:402-414

Uzhachenko R et al (2014) Fus1/Tusc2 is a novel regulator of mitochondrial calcium handling, Ca2+-coupled mitochondrial processes, and $\mathrm{Ca} 2+-$-dependent NFAT and NF-kappaB pathways in CD4+ T cells. Antioxid Redox Signal 20:1533-1547

Uzhachenko R et al (2017) Mitochondrial protein Fus1/Tusc2 in premature aging and age-related pathologies: critical roles of calcium and energy homeostasis. Aging (albany NY) 9:627-649

van Haaften-Visser DY et al (2017) Ankyrin repeat and zincfinger domain-containing 1 mutations are associated with infantile-onset inflammatory bowel disease. J Biol Chem 292:7904-7920

Vitolo OV et al (2002) Amyloid-beta -peptide inhibition of the PKA/ CREB pathway and long-term potentiation: reversibility by drugs that enhance cAMP signaling. Proc Natl Acad Sci U S A 99:13217-13221

Wang K et al (2016) Clam focal and systemic immune responses to QPX infection revealed by RNA-seq technology. BMC Genomics 17:146

Wang Y et al (2017) The mitochondrial outer membrane protein mitoNEET is a redox enzyme catalyzing electron transfer from FMNH2 to oxygen or ubiquinone. J Biol Chem 292:10061-10067

Wang Z et al (2003) Dexamethasone-induced gene 2 (dig2) is a novel pro-survival stress gene induced rapidly by diverse apoptotic signals. J Biol Chem 278:27053-27058

Warnakulasuriyarachchi D et al (2004) Translational induction of the inhibitor of apoptosis protein HIAP2 during endoplasmic reticulum stress attenuates cell death and is mediated via an inducible internal ribosome entry site element. J Biol Chem 279:17148-17157

Weller J, Budson A (2018) Current understanding of Alzheimer's disease diagnosis and treatment. F1000Res. 7
Wickham H et al (2019) Welcome to the Tidyverse. J Open Source Softw 4:1686

Wong SQ et al (2020) Autophagy in aging and longevity. Hum Genet 139:277-290

Wongkittichote P et al (2017) Propionyl-CoA carboxylase - A review. Mol Genet Metab 122:145-152

Wu M et al (2019) Identification of key genes and pathways for Alzheimer's disease via combined analysis of genome-wide expression profiling in the hippocampus. Biophys Rep 5:98-109

$\mathrm{Xu}$ D et al (2018) TPL2 kinase action and control of inflammation. Pharmacol Res 129:188-193

$\mathrm{Xu}$ J et al (2020) Ischemic Neuroprotectant PKCepsilon Restores Mitochondrial Glutamate Oxaloacetate Transaminase in the Neuronal NADH Shuttle after Ischemic Injury. Transl Stroke Res 11:418-432

Yamada M et al (2013) Rab6a releases LIS1 from a dynein idling complex and activates dynein for retrograde movement. Nat Commun 4:2033

Yamamoto-Sasaki M et al (1999) Impaired phosphorylation of cyclic AMP response element binding protein in the hippocampus of dementia of the Alzheimer type. Brain Res 824:300-303

Yamazaki H et al (1996) Cloning and characterization of KAP3: a novel kinesin superfamily-associated protein of KIF3A/3B. Proc Natl Acad Sci U S A 93:8443-8448

Yin F et al (2016a) Energy metabolism and inflammation in brain aging and Alzheimer's disease. Free Radic Biol Med 100:108-122

Yin Y et al (2016b) Tau accumulation induces synaptic impairment and memory deficit by calcineurin-mediated inactivation of nuclear CaMKIV/CREB signaling. Proc Natl Acad Sci U S A 113:E3773-E3781

Yuan XZ et al (2017) The Role of ADAM10 in Alzheimer's Disease. J Alzheimers Dis 58:303-322

Yuniati L et al (2016) Tumor suppressor BTG1 promotes PRMT1mediated ATF4 function in response to cellular stress. Oncotarget 7:3128-3143

Yuniati L et al (2019) Tumor suppressors BTG1 and BTG2: Beyond growth control. J Cell Physiol 234:5379-5389

Zhang L et al (2015) Massive expansion and functional divergence of innate immune genes in a protostome. Sci Rep 5:8693

Publisher's Note Springer Nature remains neutral with regard to jurisdictional claims in published maps and institutional affiliations. 